\title{
APPLYING THE GENETIC ALGORITHM AND THE CONSEQUENTIAL CONVEX APPROXIMATION PROGRAMMING FOR COMPOSITE STRUCTURE OPTIMIZATION
}

\author{
Nguyen Thoi Trung, Ngo Thanh Phong \\ Department of Mathematics and Informatics, \\ University of Natural Sciences-VNU-HCM
}

\begin{abstract}
This paper applies the genetic algorithm and consequential convex approximation programming to deal with problems of minimizing the strain energy of a linear elastic fiber reinforced composite laminate in a state of plane stress. The directions of fibers are used as design variables. From the numerical results, an evaluation of two optimization techniques is performed.
\end{abstract}

\section{Introduction}

Fiber reinforced composite materials are ideal for structural applications where high stiffness and strength are required at low weight. Aircraft and spacecraft are typical weight sensitive structure, in which composite materials are cost effective. To obtain the full advantage of the fiber reinforcement, fibers must be distributed and oriented optimally with respect to the actual strain field.

Hopever, due to the objective function and constrains are implicit depending on the dest $_{\text {t }}$ a variables, it is impossible to use the traditional optimization methods such as the external penalty, the internal penalty, the Lagrange multiply methods, etc ... to solve directly. We must use new methods such as the genetic algorithm and the sequential convex approximation programming to solve.

\section{Behavior theory of the composite laminate in the plane stress state $[1]$}

2.1 Elastic equation in the principle axis

$$
\left[\begin{array}{l}
\sigma_{1} \\
\sigma_{2} \\
\sigma_{6}
\end{array}\right]=\left[\begin{array}{ccc}
Q_{11} & Q_{12} & 0 \\
Q_{12} & Q_{22} & 0 \\
0 & 0 & Q_{66}
\end{array}\right]\left[\begin{array}{l}
\epsilon_{1} \\
\epsilon_{2} \\
\epsilon_{6}
\end{array}\right],
$$




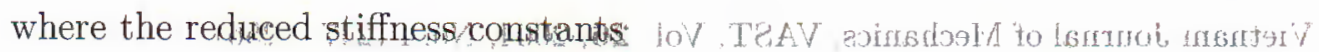

$$
Q_{11}=\frac{E_{1}}{1-\frac{E_{2}}{E_{1}} \nu_{12}^{2}}, \quad Q_{22}=\frac{E_{2}}{E_{1}} Q_{11},
$$

$$
\begin{aligned}
& \text { AH OUA Q }
\end{aligned}
$$

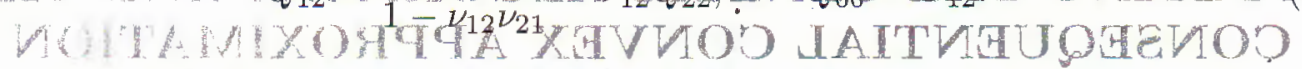

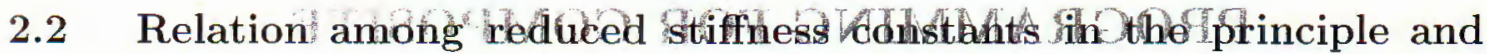

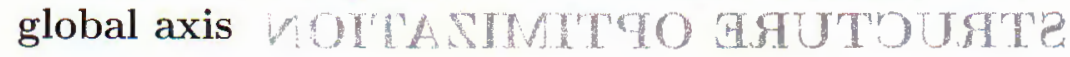

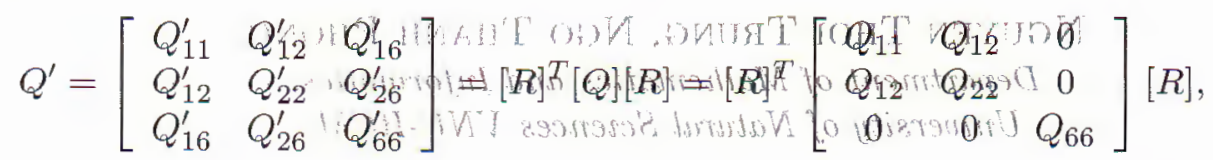

where

$$
\begin{aligned}
& Q_{i j} \text { - the reduced stiffness constants in the global axis (direction } X^{2} \text { Fig. } 1 \text { ) } \\
& Q_{i j} \text { - the reduced stiffness constants in the prindiple axis (direction } X \text { (Fig. } 1 \text { ) } \\
& \theta \text { - the angle between the direction of fiber (the principle axis) and the difection } X \\
& \text { (the global axis) (Fig. 1). }
\end{aligned}
$$

$$
[R]=\left[\begin{array}{ccc}
\cos ^{2} \theta & \sin ^{2} \theta & -2 \sin \theta \cos \theta \\
\sin ^{2} \theta & \cos ^{2} \theta & 2 \sin \theta \cos \theta \\
\sin \theta \cos \theta & -\sin \theta \cos \theta & \cos ^{2} \theta-\sin ^{2} \theta
\end{array}\right] \text { itouboutur } \quad \boldsymbol{L}
$$

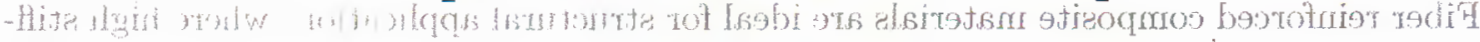

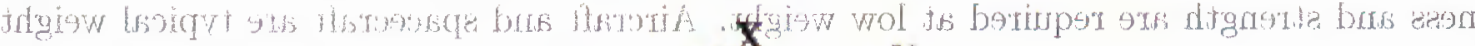

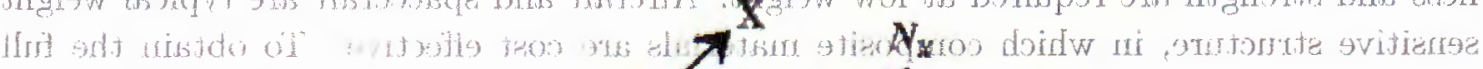

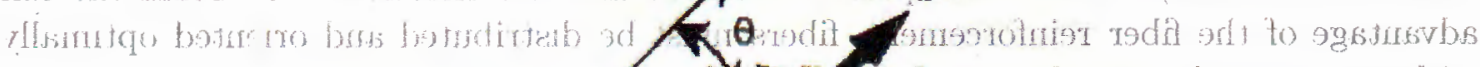

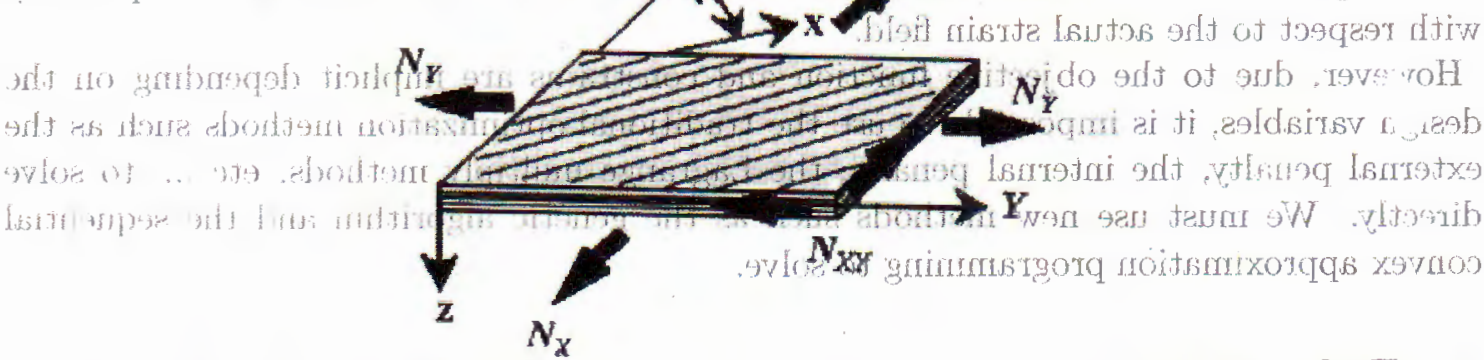

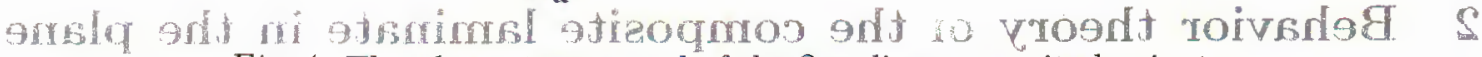
Fig. 1. The plane stress mood of the $2 n$ plies composite laminate 916 le equda

\subsection{Elastic relation and the strain 9 ergy of the $2 n_{\text {nplies }}$ composites lam-s inate}

From (2.3), we have the equation to calculates he rnatrix of reduced stiffness constants of the $2 n$ plies composite laminate:

$$
A=\left[\begin{array}{lll}
A_{11} & A_{12} & A_{16} \\
A_{21} & A_{22} & A_{26} \\
A_{16} & A_{26} & A_{66}
\end{array}\right]=\sum_{i=1}^{2 n} h_{i} *\left[\begin{array}{lll}
{ }^{\prime} Q_{11}^{\prime} & Q_{12}^{\prime} & Q_{16}^{\prime} \\
Q_{12}^{\prime} & Q_{22}^{\prime} & Q_{26}^{\prime} \\
Q_{16}^{\prime} & Q_{26}^{\prime} & Q_{66}^{\prime}
\end{array}\right]_{i},
$$


where: $h_{i}$ : the thickness of $i^{\text {th }}$ ply.

So the elastic relation of the $2 n$ plies composite laminate in the plane stress problem is of the form:

where:

$$
\left[\begin{array}{c}
N_{X} \\
N_{Y} \\
N_{X Y}
\end{array}\right]=\left[\begin{array}{lll}
A_{11} & A_{12} & A_{16} \\
A_{21} & A_{22} & A_{26} \\
A_{16} & A_{26} & A_{66}
\end{array}\right]\left[\begin{array}{c}
\epsilon_{X} \\
\epsilon_{Y} \\
\epsilon_{X Y}
\end{array}\right],
$$

$\epsilon_{i}$ - the strain vector of the $2 n$ plies composite laminate,

$N_{i}$ - the load vector of plane stress mood of $2 n$ plies composite laminate (Fig. 1), or conversely.

$$
\epsilon=\left[\begin{array}{c}
\epsilon_{X} \\
\epsilon_{Y} \\
\epsilon_{X Y}
\end{array}\right]=A^{-1}\left[\begin{array}{c}
N_{X} \\
N_{Y} \\
N_{X Y}
\end{array}\right] .
$$

The strain energy $E$ of the $2 n$ plies composite laminate is determined by:

$$
E=\frac{1}{2} \epsilon^{T} A \epsilon
$$

We can take the strain energy as the objective function of the problems of composite structure optimization.

\section{The genetic algorithm (GA) [6]}

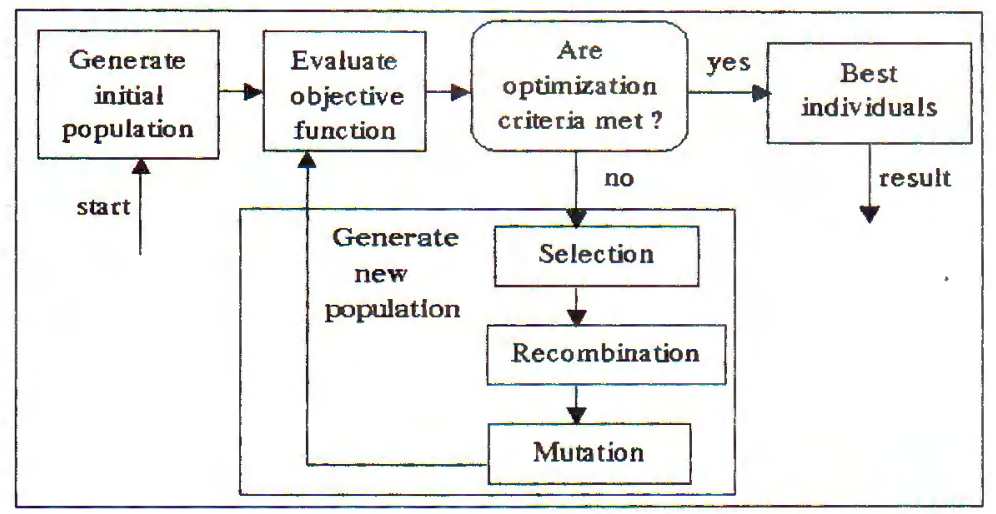

Fig. 2. Structure of a single population GA

GA is a stochastic search method that mimics the metaphor of natural biological evolution. GA operates on a population of potential solutions applying the principle of survival of the fittest to produce better and better approximations to a solution. At each generation, a new set of approximations is created by the process of selecting individuals according to their level of fitness in the problem domain and breeding them together using operators borrowed from natural genetics. This process leads to the evolution of populations of individuals that are better suited to their environment than the individuals that they were created from, just as in natural adaptation. 
At the beginning of the computation, a number of individuals are randomly initialized. The objective function is then evaluated for these individuals. The first generation is produced. If the optimization criteria are not met, the creation of a new generation starts. Individuals are selected according to their fitness for the production of offsprings. Parents are recombined to produce offsprings. All offsprings will be mutated with a certain probability. The fitness of the offspring is then computed. The offspring are inserted into the population replacing the parents, producing a new generation. This cycle is performed until the optimization criteria are reached.

\section{The sequential convex approximation programmings [2]}

The design optimization problem consists in minimizing an objective function $g_{0}(X)$ subjected to behavior constraints $g_{j}(X)$ insuring the feasibility of the structural design.

$$
\min g_{0}(X),\left\{\begin{array}{cc}
g_{j}(X) \leq g_{j}^{\max } & j=1, \ldots, m \\
X_{i}^{\min } \leq X_{i} \leq X_{i}^{\max } & i=1, \ldots, n
\end{array} .\right.
$$

The functions $g_{j}(X), j=0, \ldots, m$ are structural responses (e.g. mass, stresses, displacements, global stiffness) while the design variables $X_{i}, i=1, \ldots, n$ can be the thickness of some structural members, geometric parameters, or fiber orientations for composite structures. Their range of variation is defined by lower and upper bounds that reflect technological considerations.

The direct solution of problem (4.1) is prohibitive because of the computational cost of the structural and sensitivity analyses that have to be performed at each iteration of the optimization procedure. In the approximation concepts approach, the primary optimization problem (4.1) is replaced with a sequence of explicit approximated subproblems generated through first or second-order Taylor series expansion of the structural functions in terms of specific intermediate linearization variables, e.g. direct or reciprocal variables. The generated structural approximations, built from the information at the current design point, are often convex and separable. A dual formulation can then be used in a very efficient way for solving each explicit approximated sub-problem.

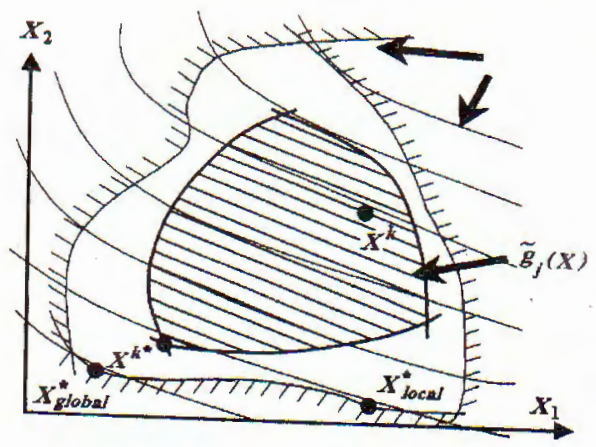

Fig. 3. Representation of problem (4.1) and its convex approximation around the current point $X^{k}$ 
The approximation concepts approach consists basically in the following step finite element analysis is performed for the initial design characterized by the values $X_{k}$ of the design variables.

From the results of the current structural analysis, that is the structural responses and the sensitivity analyses computations, an approximated optimization sub-problem is generated.

The optimization is then performed on the following sub-problem

$$
\min \tilde{\mathrm{g}}_{0}(X),\left\{\begin{array}{cc}
\tilde{\mathrm{g}}_{j}(X) \leq g_{j}^{\max } & j=1, \ldots, m \\
X_{i}^{\min } \leq X_{i} \leq X_{i}^{\max } & i=1, \ldots, n
\end{array},\right.
$$

where $\tilde{\mathrm{g}}_{j}(X)$ are the approximated structural responses. Because the approximated subproblem is fully explicit, convex and separable, it can be efficiently solved by resorting to its dual formulation.

The solution of the approximated sub-problem is adopted as a new starting point in the design space, and the optimization process is continued until convergence is achieved.

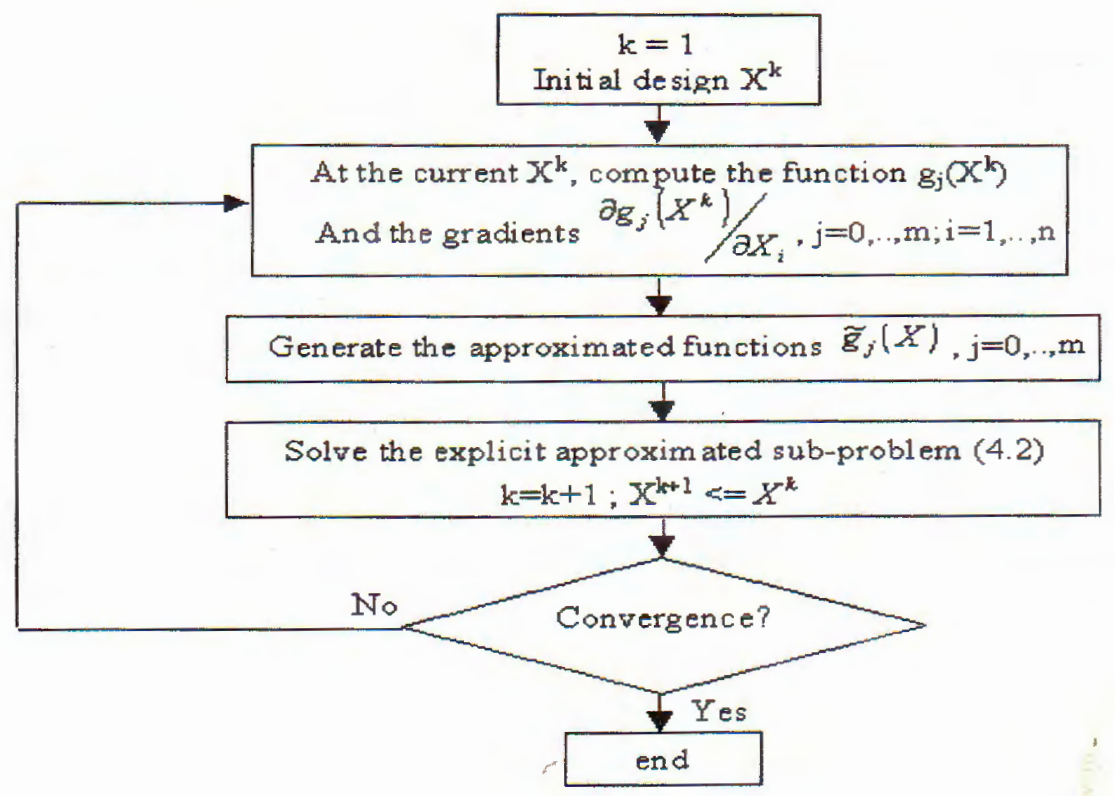

Fig. 4. Iterative scheme of the optimization using the appruximation concepts

Approximations of the MMA family (Method of Moving Asymptotes [3][4])

\subsection{Monotonous approximations}

Conlin scheme is a convex approximation based on the first order Taylor series expansion in terms of direct and reciprocal design variables. The approximation of a design function $\tilde{\mathrm{g}}_{j}(X)$ is computed based on the function value and on the first derivatives at the current 
design point $X^{k}$.

$$
\tilde{\mathrm{g}}_{j}(X)=g_{j}\left(X^{k}\right)+\sum_{+, i} \frac{\partial g_{j}\left(X^{k}\right)}{\partial X_{i}}\left(X_{i}-X_{i}^{k}\right)-\sum_{-, i}\left(X_{i}^{k}\right)^{2} \frac{\partial g_{j}\left(X^{k}\right)}{\partial X_{i}}\left(\frac{1}{X_{i}}-\frac{1}{X_{i}^{k}}\right) .
$$

The symbols $\sum_{+, i}$ and $\sum_{-, i}$ denote the summations over terms having positive and negative first order derivatives.

In the method of Moving Asymptotes or MMA, Svanberg (1987) generalized Conlin scheme by introducing two sets of new parameters, the lower and upper asymptotes, $L_{i}^{k}$ and $U_{i}^{k}$, in order to adjust the convexity of the approximation in accordance with the problem under consideration.

$$
\tilde{\mathrm{g}}_{j}(X)=g_{j}\left(X^{k}\right)+\sum_{+, i}^{n} p_{i j}^{k}\left(\frac{1}{U_{i}^{k}-X_{i}}-\frac{1}{U_{i}^{k}-X_{i}^{k}}\right)+\sum_{-, i}^{n} q_{i j}^{k}\left(\frac{1}{X_{i}-L_{i}^{k}}-\frac{1}{X_{i}^{k}-L_{i}^{k}}\right) .
$$

In (4.4) only one of the two coefficients $p_{i j}^{k}$ or $q_{i j}^{k}$ is different from zero at the same time for one design variable:

$$
\overrightarrow{p_{i j}^{k}}=\max \left\{0,\left(U_{i}^{k}-X_{i}^{k}\right)^{2} \frac{\partial g_{j}\left(X^{k}\right)}{\partial X_{i}}\right\}, q_{i j}^{k}=\max \left\{0,-\left(X_{i}^{k}-L_{i}^{k}\right)^{2} \frac{\partial g_{j}\left(X^{k}\right)}{\partial X_{i}}\right\} .
$$

So for each design variable $X_{i}$, only one asymptote, either $L_{i}^{k}$ or $U_{i}^{k}$, is used in the approximation according to the design of the first derivative $\partial g_{j}\left(X^{k}\right) / \partial X_{i}$. Therefore, the approximation is monotonous, what ever can be the real behavior of the response function. From one iteration to another, the $n$ asymptotes $L_{i}^{k}$ and $U_{i}^{k}$ are updated according to heuristic rules $(4.6)$ :

$$
L_{i}^{k}=X_{i}^{k}-s_{i}\left(X_{i}^{k}-L_{i}^{k-1}\right) ; U_{i}^{k}=X_{i}^{k}+s_{i}\left(U_{i}^{k-1}-X_{i}^{k-1}\right)
$$

proposed by Svanberg (1987), where the parameter $s_{i}$ is computed based on the variation of the corresponding design variable values $X_{i}$ within 3 iteration steps:

For two first iteration steps:

$$
s_{i}=0.5 \Rightarrow\left\{\begin{array}{c}
L_{i}^{k}=X_{i}^{k}-0.5\left(X_{i}^{\max }-X_{i}^{\min }\right) \\
U_{i}^{k}=X_{i}^{k}+0.5\left(X_{i}^{\max }-X_{i}^{\min }\right)
\end{array} .\right.
$$

For the third iteration step:

$$
s_{i}=\left\{\begin{array}{cl}
0.7 & \text { if }\left(X_{i}^{k}-X_{i}^{k-1}\right)\left(X_{i}^{k-1}-X_{i}^{k-2}\right)<0 \\
1.2 & \text { if }\left(X_{i}^{k}-X_{i}^{k-1}\right)\left(X_{i}^{k-1}-X_{i}^{k-2}\right)>0 \\
1 & \text { if }\left(X_{i}^{k}-X_{i}^{k-1}\right)\left(X_{i}^{k-1}-X_{i}^{k-2}\right)=0
\end{array}\right.
$$

\subsection{Non-monotonous approximations [3][4]}

In Svanberg (1995a), the author derived a Globally Convergent version of the Method of Moving Asymptotes 1 (GCMMA1):

$$
\tilde{\mathrm{g}}_{j}(X)=g_{j}\left(X^{k}\right)+\sum_{i=1}^{n} p_{i j}^{k}\left(\frac{1}{U_{i}^{k}-X_{i}}-\frac{1}{U_{i}^{k}-X_{i}^{k}}\right)+\sum_{i=1}^{n} q_{i j}^{k}\left(\frac{1}{X_{i}-L_{i}^{k}}-\frac{1}{X_{i}^{k}-L_{i}^{k}}\right) .
$$


This is an extension of the MMA scheme (4.4), where both $p_{i j}^{k}$ and $q_{i j}^{k}$ are simultaneously non-zero, which means that now both $L_{i}^{k}$ and $U_{i}^{k}$ are used at the same time to generate the approximation (4.9). This leads to the non-monotonous character of the approximation as illustrated in Fig. 5 .

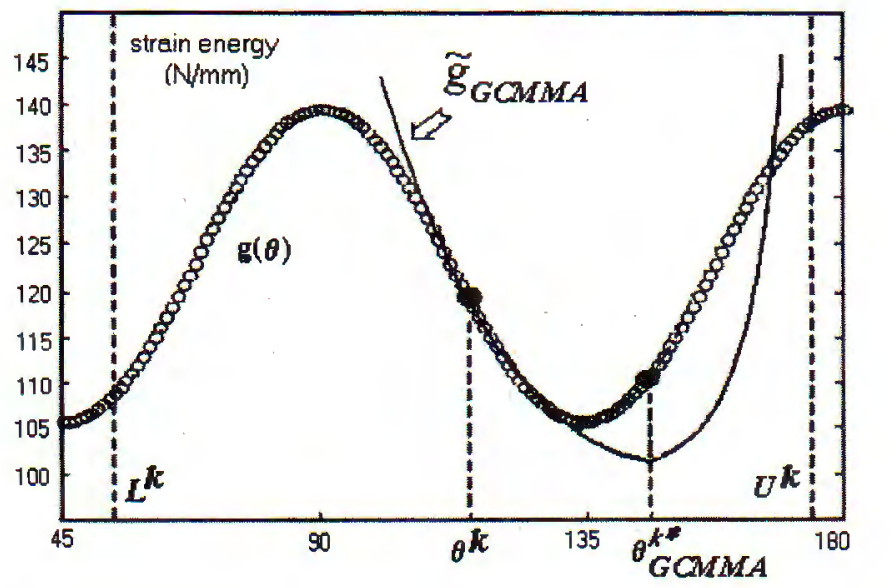

Fig. 5. Approximation of the strain energy $g(\theta)$ for optimal orientation in a single ply laminate.

A non-monotonous approximation is advised

At the stage $k$ of the optimization process, GCMMA1 is defined by the function value $g_{j}\left(X^{k}\right)$, the parameters $p_{i j}^{k}$ and $q_{i j}^{k}$ computed based on the first order derivatives, on a non-monotonic parameter $\rho_{i}^{k}$, and by the $n$ pairs of asymptotes $L_{i}^{k}$ and $U_{i}^{k}$.

$$
\begin{gathered}
\text { if } \frac{\partial g_{j}\left(X^{k}\right)}{\partial X_{i}}<0 \Rightarrow\left\{\begin{array}{c}
p_{i j}^{k}=\left(U_{i}^{k}-X_{i}^{k}\right)^{2} \frac{\rho_{j}^{k}}{2}\left(U_{i}^{k}-L_{i}^{k}\right) \\
q_{i j}^{k}=\left(X_{i}^{k}-L_{i}^{k}\right)^{2}\left(-\frac{\partial g_{j}\left(X^{k}\right)}{\partial X_{i}}+\frac{\rho_{j}^{k}}{2}\left(U_{i}^{k}-L_{i}^{k}\right) .\right.
\end{array}\right) \\
\text { if } \frac{\partial g_{j}\left(X^{k}\right)}{\partial X_{i}}>0 \Rightarrow\left\{\begin{array}{c}
p_{i j}^{k}=\left(U_{i}^{k}-X_{i}^{k}\right)^{2}\left(\frac{\partial g_{j}\left(X^{k}\right)}{\partial X_{i}}+\frac{\rho_{j}^{k}}{2}\left(U_{i}^{k}-L_{i}^{k}\right)\right) \\
q_{i j}^{k}=\left(X_{i}^{k}-L_{i}^{k}\right)^{2} \frac{\rho_{j}^{k}}{2}\left(U_{i}^{k}-L_{i}^{k}\right)
\end{array}\right.
\end{gathered}
$$

The non-monotonic parameter and the asymptotes are updated according to rules given in Svanberg $(1987,1995 \mathrm{a})$ that insures the global convergence property of the approximation scheme.

For the first iteration step:

$$
\rho^{0}=\frac{1}{5 n} \sum_{i=1}^{n} \frac{1}{X_{i}^{\max }-X_{i}^{\min }}\left|\frac{\partial g\left(X^{0}\right)}{\partial X_{i}}\right| .
$$

For the $k^{\text {th }}$ iteration step:

$$
\left\{\begin{array}{ccc}
\rho^{k}=2 \rho^{k-1} & \text { if } & \tilde{g}^{k-1}\left(X^{k}\right)<g\left(X^{k}\right) \\
\rho^{k}=\rho^{k-1} & \text { if } & \tilde{\mathrm{g}}^{k-1}\left(X^{k}\right) \geq g\left(X^{k}\right)
\end{array} .\right.
$$

$\rho$ must $>0$. When $\rho_{j}^{k}=0$, one resorts to the monotonous MMA approximation. 


\section{The numerical solution [5]}

The objective function to be minimized is the strain energy of homogeneous symmetric laminates including $2 * 3$ plies subjected to restrictions with only the constraints of bound of fiber orientation (lower and upper bounds). Here there are not the behavior constraints $g_{j}(\theta)$. The fiber orientations are the design variables. The thickness of each plies is constant and equal to $t=1 \mathrm{~mm}$. The laminate is in a state of plane stress.

The design optimization problem is presented in such that:

$$
\min \frac{1}{2} \epsilon^{T} A \epsilon(\mathrm{N} / \mathrm{mm}) 0 \leq \theta_{i} \leq 1801 \leq i \leq 3
$$

The data of the problem:

$E_{x}=1.8 E 5\left(\mathrm{~N} / \mathrm{mm}^{2}\right):$ Elastic module in axis $x$

$E_{y}=0.8 E 4\left(\mathrm{~N} / \mathrm{mm}^{2}\right)$ : Elastic module in axis $y$

$n_{x y}=0.3:$ Poisson factor

$G_{12}=0.6 E 4\left(\mathrm{~N} / \mathrm{mm}^{2}\right):$ Shear module

$N_{X}=37.50(\mathrm{~N} / \mathrm{mm}):$ The normal force in the axis $X$

$N_{Y}=2500(\mathrm{~N} / \mathrm{mm}):$ The normal force in the axis $Y$

$N_{X Y}=5000(\mathrm{~N} / \mathrm{mm}):$ The force in the axis $X Y$

$\mathrm{th}=\left[\theta_{1}, \theta_{2}, \theta_{3}, \theta_{3}, \theta_{2}, \theta_{1}\right]:$ The 3 -variable problem

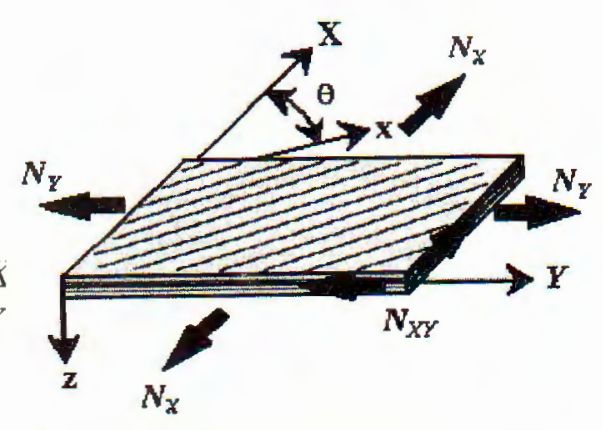

Fig. 6. The plane stress problem

\section{The results}

\begin{tabular}{|c|c|c|}
\hline The 3-variable problem & GA & GCMMA1 \\
\hline The initial fiber orientations & & $\theta_{1}=60 ; \theta_{2}=20 ; \theta_{3}=110$ \\
\hline The number of iteritions & & 17 \\
\hline $\begin{array}{c}\text { The number of calculating } \\
\text { the objective function }\end{array}$ & 16020 & 72 \\
\hline The optimal fiber orientation & $\theta_{1}=41.4375$ & $\theta_{1}=42.5794$ \\
& $\theta_{2}=41.4375$ & $\theta_{2}=40.5936$ \\
& $\theta_{3}=131.4374$ & $\theta_{3}=131.4599$ \\
\hline The strain energy (N/mm) & 50.5637 & 50.6057 \\
\hline The solving time (second) & 34.87 & 9.01 \\
\hline
\end{tabular}

We apply the sequential convex approximation programming GCMMAI by letting $\theta_{i}=X_{i}, \theta_{i}^{\min }=X_{i}^{\min }=0$, and $\theta_{i}^{\max }=X_{i}^{\max }=180$. Then we give the design variables (fiber orientations) initial values $\theta_{i}$, and calculate the objective function $g_{0}\left(\theta^{k}\right)=\frac{1}{2} \epsilon^{T} A \epsilon$ and the first derivatives $\partial g_{j}\left(\theta^{(k)}\right) / \partial X_{i}$. Then we use (4.6) (4.7) (4.8) to calculate $L_{i}^{k}, U_{i}^{k}$; use (4.12) (4.13) to calculate $\rho^{i}$; use (4:10) (4.11) to calculate $p_{i j}^{k}, q_{i j}^{k}$. Then we use (4.9) to built the approximated function $\tilde{\mathrm{g}}_{j}(\theta)$. Then we use traditional optimization methods to calculate the new fiber orientations which are adopted as a new starting point in the design space, and the optimization process is continued until convergence is achieved. 

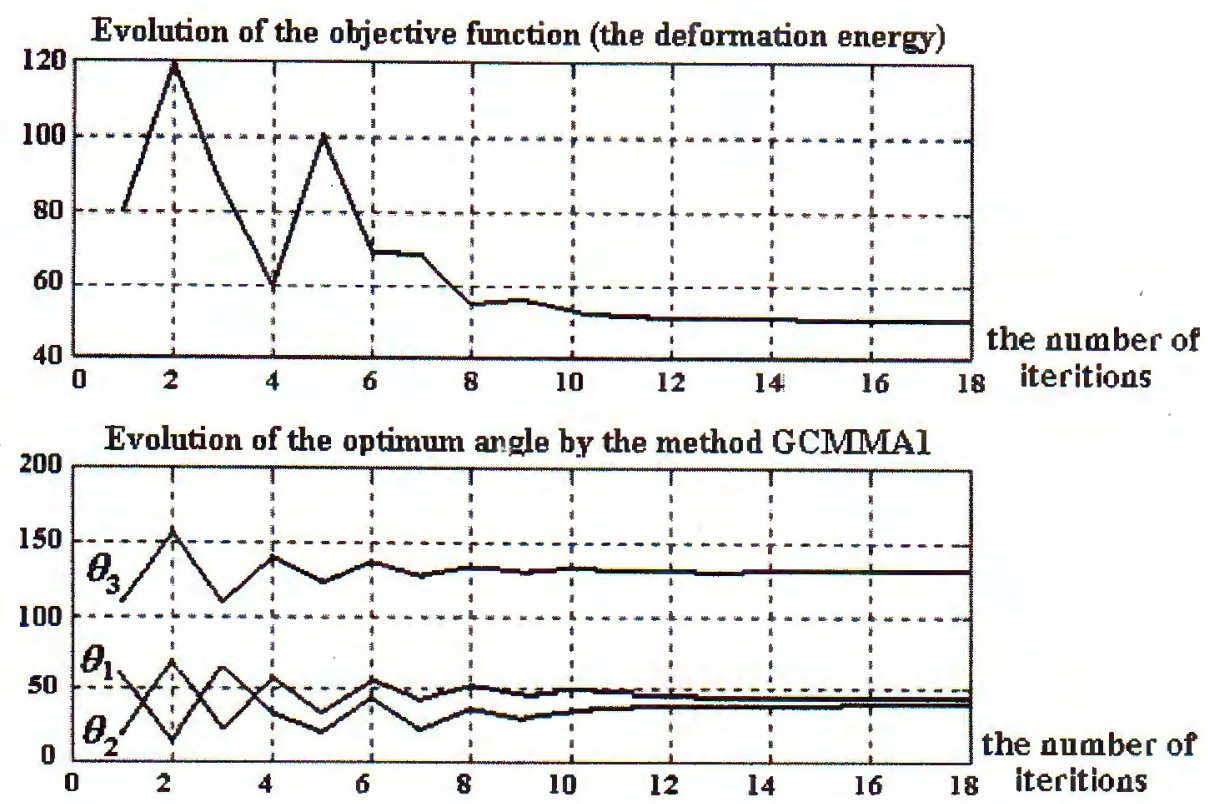

Fig. 7. The results of the 3-variable problem by the method GCMMA1

\section{Comments}

GA gives the best result of the objective function (the lowest strain energy $=50.5637$ $\mathrm{N} / \mathrm{mm}$ ). The solving time of GCMMA1 (9.01 seconds) is very much faster than of GA (34.87 seconds).

The number of calculating the objective function of GA (16020) is too big compared with of the method GCMMA1 (72). So GA will take a very long time to solve the complicated problems which require building and solving finite element in each iteration to calculate the objective function.

\section{Conclusion}

This paper has shown how to apply GA and GAMMA1 for composite structure optimization.

From the results of the numerical solution, we can conclude about two different optimization techniques in the frame of the design of composite structures in following:

GA: is a general method to solve the optimization problem in many fields. This algorithm gives the better results than the sequential convex approximation programmings (the strain energy is lower). However, due to its general character, this method requires the long time to solve.

GCMMA1: This method also give the exact results. Although, these results are a little worse than those of GA, but the solving time of the sequential convex approximation programming is very much faster than that of GA. So, this method is very suitable to solve the big optimization problems.

The study is implemented partly with the financial support from the Vietnam National Council of Natural Sciences. 


\section{References}

1. Berthelot J. M., Materiaux Composites, Ed. Masson, 1992.

2. Michael Bruyneel and Claude Fleury, Composite structures Optimization using Sequential Convex Programming, Computational Techniques for Laminates, Composites on Computational Structures Technology, Leuven, Belgium, 2000.

3. Michael Bruyneel, Pierre Duysinx, and Claude Fleury, A Family of MMA Approximations for Structural Optimization, Universite de Liege, 2001.

4. Muriel Beckers, Optimisation de Structures en Variables Discretes, These de Doctorat, Universite de Liege, 1997.

5. Nguyen Thoi Trung, Fibers orientation optimization in laminates by using the genetic algorithm and the sequential convex approximation programming, Thesis of Master, University of Natural Sciences - VNU-HCM (in Vietnamese), 2003.

6 The theory of the genetic algorithm, http://www.systemtechnik.tu-ilmenau.de/ pohlheim/GA-Toolbox/algintro.html.

Received January 8, 2004

\section{ÁP DƯNG GIẢI THUẬT DI TRUYỀN VÀ QUY HOẠCH XẤP Xİ LỒI TUẦN TỰ CHO BÀI TOÁN TỐI ƯU HÓA CẤU TRỨC COMPOSITE}

Bài báo áp dụng giải thuật di truyền và quy hoạch xấp xỉ lồi tuần tự để giải bài toán cực tiểu năng lượng biến dạng của tấm lamiate composite được gia cường sợi đàn hồi tuyến tính trong trạng thái ứng suất phẳng. Biến thiết kế là sợi của các lớp. Từ kết quả số, đã đưa ra nhận xét đánh giá hai phương pháp. 